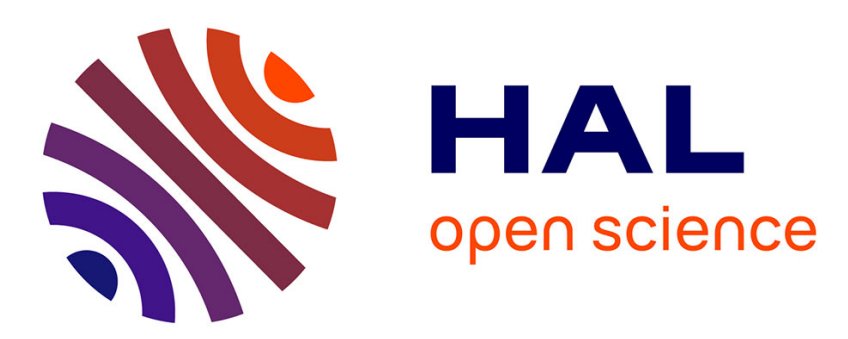

\title{
Applications de techniques nouvelles à la construction des machines électrostatiques. - Un exemple: le VIVITRON
}

\author{
F. Haas
}

\section{To cite this version:}

F. Haas. Applications de techniques nouvelles à la construction des machines électrostatiques.

- Un exemple: le VIVITRON. Revue de Physique Appliquée, 1988, 23 (9), pp.1431-1439. 10.1051/rphysap:019880023090143100 . jpa-00245967

\section{HAL Id: jpa-00245967 https://hal.science/jpa-00245967}

Submitted on 1 Jan 1988

HAL is a multi-disciplinary open access archive for the deposit and dissemination of scientific research documents, whether they are published or not. The documents may come from teaching and research institutions in France or abroad, or from public or private research centers.
L'archive ouverte pluridisciplinaire HAL, est destinée au dépôt et à la diffusion de documents scientifiques de niveau recherche, publiés ou non, émanant des établissements d'enseignement et de recherche français ou étrangers, des laboratoires publics ou privés. 


\title{
Applications de techniques nouvelles à la construction des machines électrostatiques. Un exemple : le VIVITRON
}

\author{
F. Haas \\ Centre de Recherches Nucléaires (IN2P3), 67037 Strasbourg Cedex, France
}

(Reçu le 15 octobre 1987, accepté le 17 mai 1988)

\begin{abstract}
Résumé. - Le VIVITRON est un accélérateur électrostatique du type Tandem de 35 Millions de Volts actuellement en cours de construction au Centre de Recherches Nucléaires de Strasbourg. Les nouvelles techniques de montage du générateur haute tension sont décrites. Dans la conception de la nouvelle machine, l'accent a été mis sur le problème d'une meilleure «gestion» de l'énergie stockée. Plusieurs applications futures de ce type d'accélérateur sont évoquées.
\end{abstract}

Abstract. - The VIVITRON is an electrostatic Tandem accelerator of 35 Megavolts actually under construction at the Strasbourg Centre de Recherches Nucléaires. The novel techniques used to build the high voltage generator are described. It is shown that the major improvement of the new machine consists in a «safer » treatment of the stored energy. Future applications of this kind of accelerators are briefly presented.

\section{Introduction.}

Les machines électrostatiques sont certainement les accélérateurs de particules les plus performants dans la plage d'énergies de bombardement qu'elles permettent de couvrir. Les accélérateurs électrostatiques du type Tandem ont permis au cours de ces 25 dernières années de faire des découvertes importantes en physique nucléaire grâce aux réactions induites par ions lourds. Dans ce contexte, il était donc tout à fait justifié que plusieurs laboratoires se soient engagés dans la réalisation de machines plus performantes au point de vue voltage $(V>20 \mathrm{MV})$. Il faut cependant constater qu'à l'heure actuelle, ces accélérateurs fonctionnent à des tensions très inférieures aux tensions nominales.

Le projet VIVITRON (VIV) du Centre de Recherches Nucléaires de Strasbourg est encore plus ambitieux. L'objectif de $35 \mathrm{MV}$ que nous nous sommes fixé, nécessite la mise en œuvre de techniques nouvelles de construction qui concernent principalement le générateur haute tension. Nous pensons que ces développements originaux décrits dans le présent article et proposés par M. Letournel sont susceptibles de déboucher sur une nouvelle génération de machines électrostatiques ce qui présente un grand intérêt pour les utilisateurs futurs de ce type d'accélérateurs.

\section{Le point sur les accélérateurs électrostatiques Tandem dans le monde.}

Il est bien connu qu'un accélérateur électrostatique délivre un faisceau de très grande qualité: Pourtant le succès en physique nucléaire du Tandem comme machine à ions lourds est dû aussi aux deux facteurs suivants :

- le développement des sources d'ions négatifs à «sputtering». Dans le VIV par exemple, il est envisagé d'accélérer une soixantaine de faisceaux de nature différente ;

- les possibilités d'épluchage des ions accélérés traversant des milieux solides ou gazeux.

L'évolution des performances en énergie de l'accélérateur Tandem est marquée par quelques étapes importantes :

- la construction en 1955 par Van de Graaff et la compagnie HVEC (USA) du premier Tandem ;

- la mise en service durant les années 60 de nombreuses machines fonctionnant à des voltages $V$ inférieurs à $10 \mathrm{MV}$; 
- la mise en service durant les années 70 d'une douzaine de Tandems MP avec $V \simeq 10 \mathrm{MV}$ et dont il existe deux exemplaires en France à l'IPN d'Orsay et au CRN de Strasbourg ;

- la mise en service au cours de ces dernières années, de super-Tandems susceptibles d'atteindre des tensions supérieures à $15 \mathrm{MV}$.

Cette dernière catégorie comprend un nombre restreint de machines installées à Oak Ridge (U.S.A.), Daresbury (G.B.), Jaeri (Japon) et Legnaro (Italie) qui ont atteint à l'heure actuelle les tensions maximum de fonctionnement suivantes: 22, 19, 17 et $16 \mathrm{MV}$, respectivement. Le Tandem MP de Strasbourg figure également dans cette catégorie puisqu'à la suite de plusieurs opérations de montée en tension basées sur des modifications originales proposées par le CRN et effectuées sur le tube accélérateur et le générateur, la tension de la machine a été progressivement augmentée de 10 à
18 MV entre 1972 et 1982. D'ailleurs, les différentes modifications mises en œuvre et testées avec succès sur le Tandem MP ont été incorporées dans la conception du VIV.

Le point important à noter est que les deux superTandems à Oak Ridge et Daresbury fonctionnent actuellement à des tensions de 22 et 19 MV qui sont à comparer aux tensions nominales de 25 et $30 \mathrm{MV}$, respectivement. Avant de discuter de ce problème crucial de limitation en tension, il faut mentionner que pour atteindre des énergies plus élevées, plusieurs laboratoires possédant des Tandems ont choisi des solutions à base de post-accélérateur où le Tandem sert d'injecteur à un cyclotron ou à un accélérateur linéaire.

\section{Limitation en tension dans les super-Tandems.}

La question fondamentale qui se pose est donc de savoir d'où vient cette limitation en tension. L'expé-

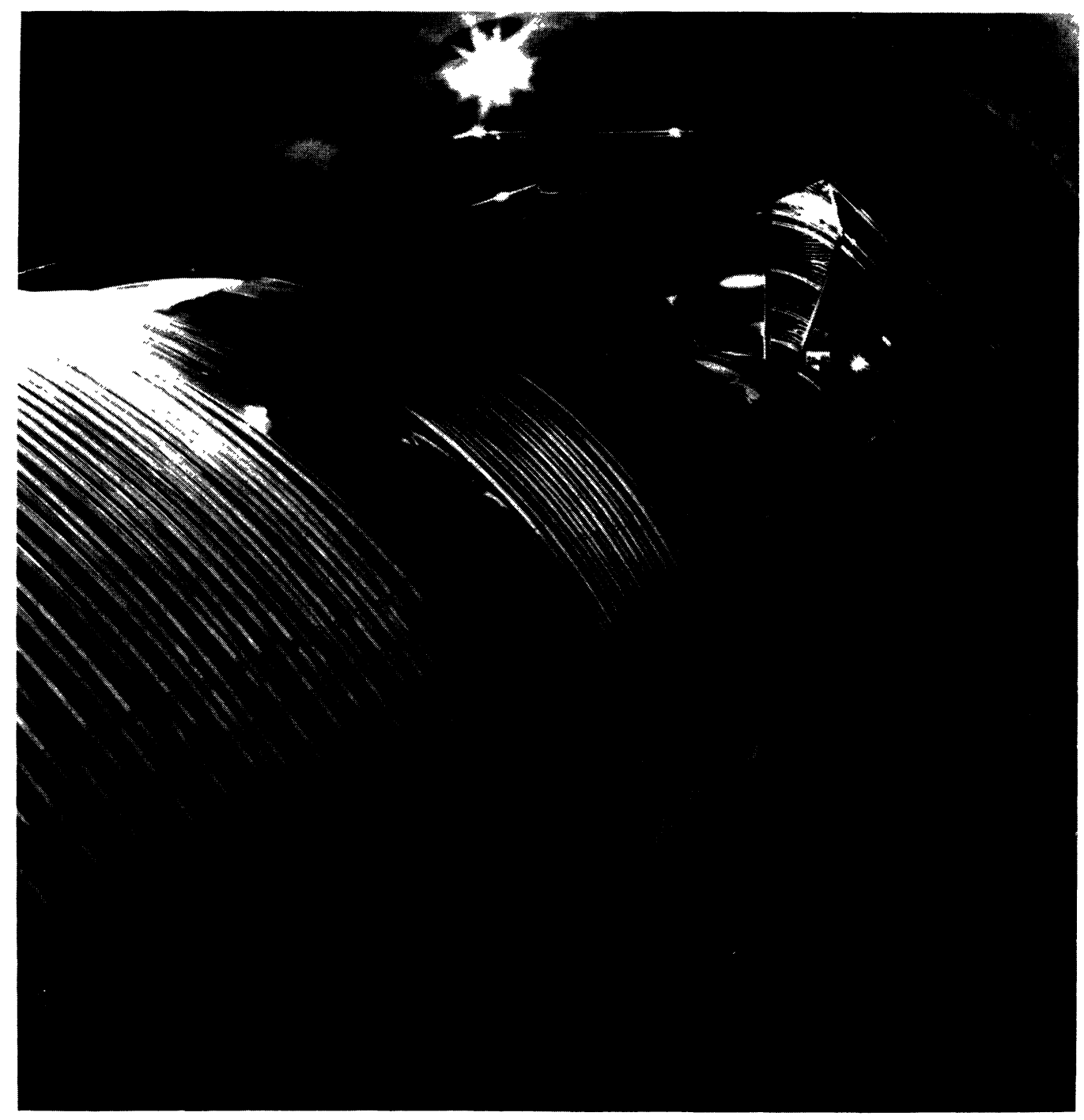

Fig. 1. - Cage à électrodes discrètes installée dans le Tandem MP du CRN depuis 1982.

[Cage of discrete electrodes installed in the Strasbourg Tandem since 1982.] 
rience montre que dans une machine électrostatique le déclenchement de décharges est un phénomène quasiment inévitable. Nous avons démontré que dans la plupart des cas, l'origine des décharges se situait à l'intérieur du tube qui est le siège de processus dynamiques (ce qui contraste avec le qualificatif de «statique » attribué à la machine) liés au vide, au champ entre électrodes, à la géométrie et à la nature des électrodes, aux parties isolantes du tube, à l'émission de particules secondaires... Tous ces problèmes sont d'une très grande importance pour le fonctionnement des accélérateurs électrostatiques, ils ne peuvent pas être discutés dans le contexte de cet article mais mériteraient certainement des réflexions et des études plus poussées. Dans une machine «classique », le champ entre l'électrode centrale et l'électrode extérieure (le réservoir) est en $1 / R$ où $R$ est la distance à partir du centre. Pour une telle configuration, la répartition de l'énergie stockée n'est pas uniforme mais concentrée autour de l'électrode centrale. En cas de décharges, cette énergie se dissipe et il arrive fréquemment que des organes vitaux de l'appareil soient touchés. On sait que le tube est l'élément clé de l'accélérateur mais placé dans l'axe central de la machine c'est aussi un élément exposé et fragile. Dans pratiquement tous les Tandems et a fortiori dans les super-Tandems, ce sont les conséquences des décharges qui sont la cause d'une grande partie $(\sim 50 \%)$ des périodes d'arrêt machine.

A l'heure actuelle, la tension maximum atteinte sur un Tandem en fonctionnement est de $22 \mathrm{MV}$ (à Oak Ridge), pour le VIV il est prévu une tension nominale de $35 \mathrm{MV}$. Il est donc évident que le problème majeur avec lequel nous serons confrontés est celui du «traitement » de l'énergie stockée.

\section{Solutions proposées pour le VIVITRON.}

Pour une tension de 35 MV sur l'électrode terminale, l'énergie stockée dans la nouvelle machine sera de $\sim 440 \mathrm{~kJ}$. Dans la conception du VIV, notre objectif principal était de tendre vers une répartition aussi uniforme que possible de cette énergie à l'intérieur du réservoir. Il fallait donc, contrairement aux machines classiques où le champ est en $1 / R$, générer un champ électrique quasi constant entre l'électrode centrale et l'électrode extérieure (réservoir). La solution proposée consiste à placer dans l'espace interélectrode principal des électrodes intermédiaires à caractère « discret ». Ces électrodes se présentent sous la forme de 7 portiques (ou cages) à 7 bras. La figure 1 montre le portique installé dans le Tandem MP et qui nous a permis une montée en tension à $18 \mathrm{MV}$ pour cette machine. Différentes vues de portiques du VIV sont présentées sur les figures 2 et 3.

Dans le cadre de cet article, la description du VIV se limitera au générateur haute tension. La figure 4 représente une vue schématique de l'intérieur du nouvel accélérateur. Les aspects nouveaux de la machine concernent essentiellement le générateur et, plus particulièrement, les points relatifs aux répartitions du potentiel et à l'isolation que nous allons maintenant passer en revue brièvement.

RÉPARTITION DU POTENTIEL. - Le potentiel est réparti longitudinalement à l'aide d'électrodes

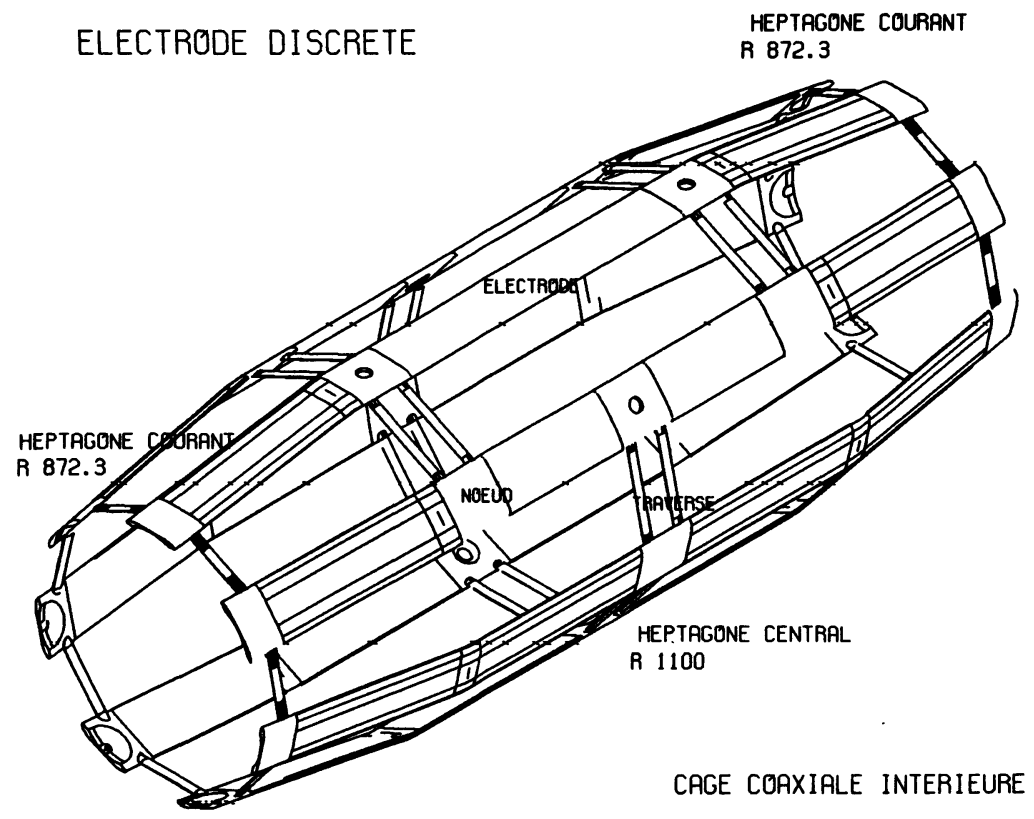

Fig. 2. - Dessin obtenu par CAO de la cage coaxiale intérieure à électrodes discrètes.

[View of the most central coaxial cage of discrete electrodes.] 


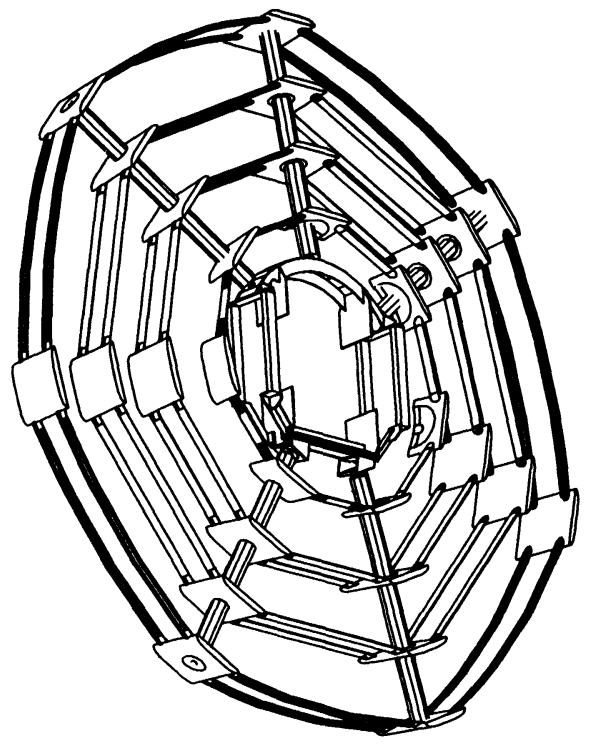

Fig. 3. - Dessin obtenu par CAO d'une coupe transversale de l'espace intérieur du générateur VIVITRON au niveau d'une section morte.

[Cross section of the VIVITRON generator at the level of a dead section.]

colonne (Fig. 4) associées à des chaînes de résistances. La forme des électrodes est conçue pour protéger électriquement le noyau central qui comprend le tube. Le potentiel est réparti radialement par l'intermédiaire des 7 portiques à électrodes discrètes (Fig. 4). Le champ électrique entre l'électrode centrale et le réservoir est pratiquement constant. Par rapport à une machine classique et pour une même tension, l'utilisation des électrodes intermédiaires a permis de réduire les dimensions radiales d'un facteur 2 .

IsOLATION. - L'isolation longitudinale est obtenue à l'aide d'isolants de grande longueur $(\sim 2,8 \mathrm{~m})$ qui se présentent sous la forme de planches découpées

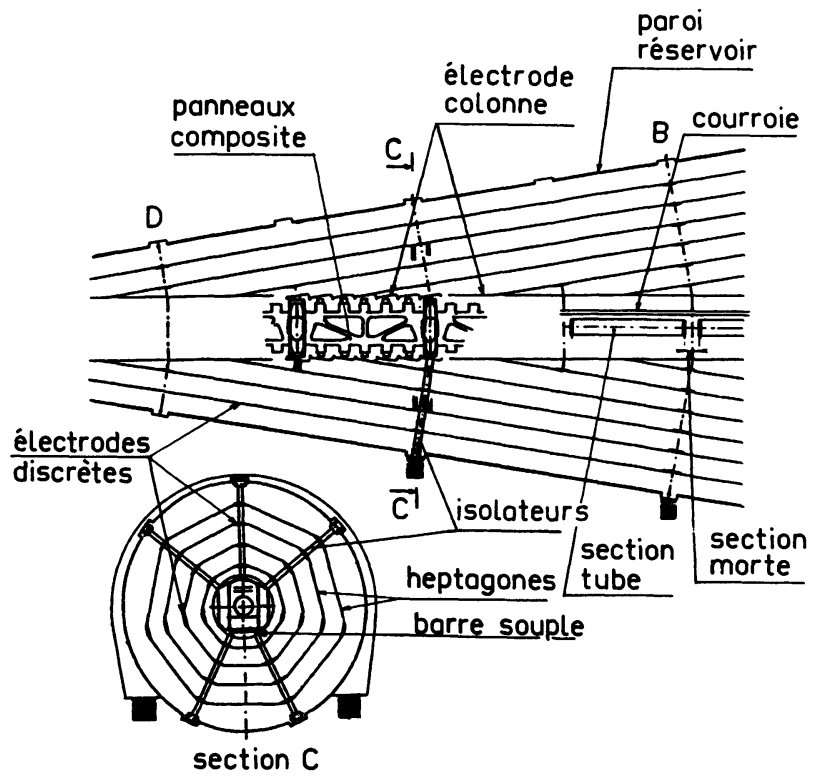

Fig. 5. - Aspects mécaniques du VIVITRON : schéma de la solution proposée pour le support des éléments à l'intérieur du réservoir.

[Mechanical aspects of the VIVITRON : scheme of the proposed solution to support the elements inside the tank.]

(Figs. 5 et 6) en matériau composite constitué de fibres de verre enrobées dans de la résine époxyde. L'utilisation de ce matériau, placé dans un champ électrique maximum de $20 \mathrm{kV} / \mathrm{cm}$, allège considérablement le poids de la partie longitudinale de la structure (réduction d'un facteur 10) et nous a permis la construction d'une machine horizontale $(51 \mathrm{~m})$ pour laquelle une structure portante radiale est construite à partir du réservoir (Fig. 5). Elle est constituée par des empilements d'isolateurs placés entre les portiques à électrodes discrètes. Les isolateurs (plots isolants) sont en résine époxyde chargée d'alumine et de silice. Ils sont soumis à un champ

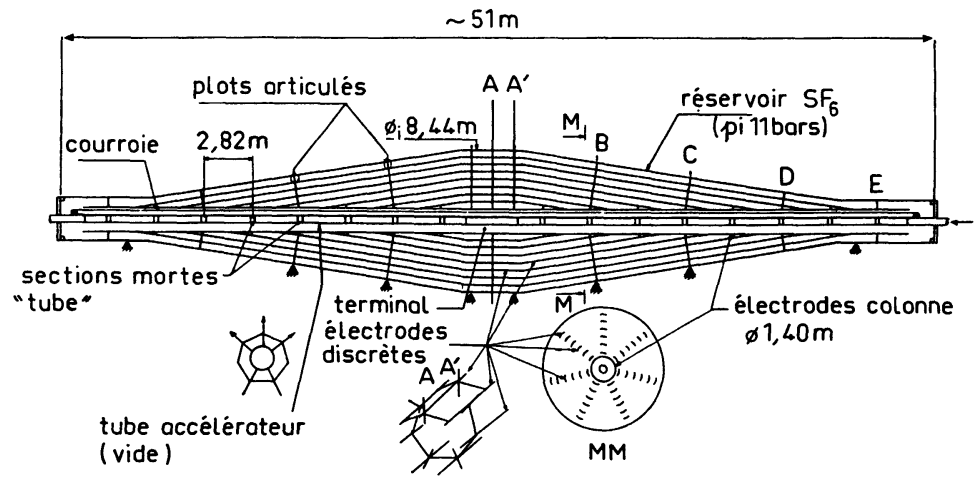

Fig. 4. - Vue schématique du VIVITRON montrant les éléments de base de la nouvelle machine.

[Schematic view of the VIVITRON showing the basic elements of the new machine.] 


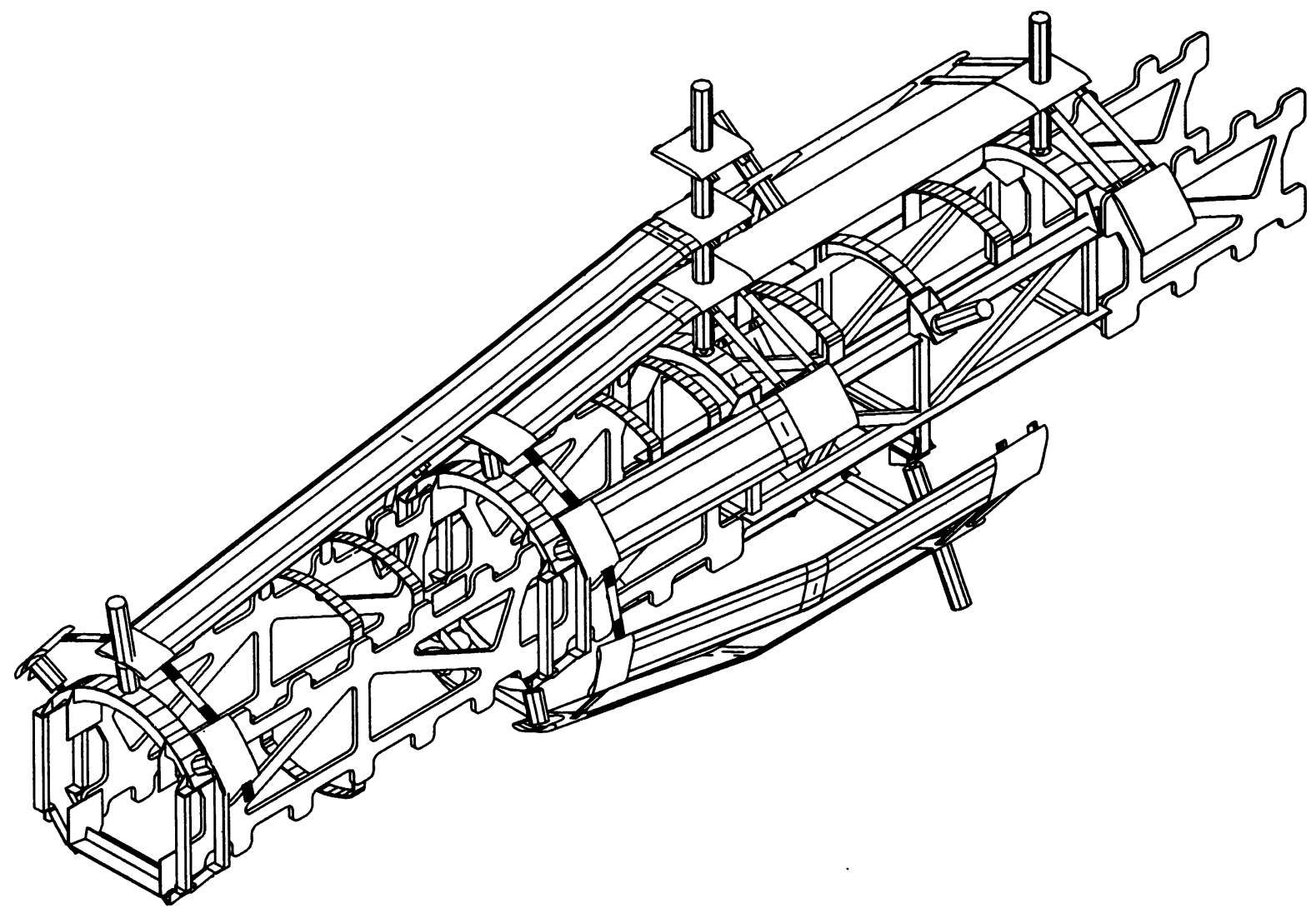

Fig. 6. - Dessin obtenu par CAO de la partie centrale du VIVITRON.

[View of the VIVITRON central part.]

moyen maximum de $120 \mathrm{kV} / \mathrm{cm}$ ce qui correspond à une tension de 4,5 MV. Le plot, de longueur $40 \mathrm{~cm}$ et de diamètre $12 \mathrm{~cm}$ (Fig. 7), est un élément essentiel du VIV tant du point de vue mécanique qu'électrique (isolation) et ceci d'autant plus que leur nombre est élevé $(N=248)$. Ce type d'isolateur a été développé pour le projet dans le cadre d'une collaboration avec C. M. Cooke (MIT) qui travaille depuis de nombreuses années sur le développement et l'utilisation d'isolateurs dans les lignes souterraines haute tension.

Toutes les nouvelles techniques de construction ainsi que les matériaux choisis ont été testés dans le Tandem MP ou auprès d'un générateur du type VIV spécialement conçu et construit à cet effet (Fig. 8).

Nous compléterons cette description du VIV en indiquant quelques autres caractéristiques de la nouvelle machine :

volume du réservoir : $1200 \mathrm{~m}^{3}$; longueur : $51 \mathrm{~m}$; diamètre (centre) : $8,5 \mathrm{~m}$;

diamètre (extrémités): 2,6 m ; épaisseurs de l'acier : $38 \mathrm{~mm}$ au centre, $16 \mathrm{~mm}$ aux extrémités.

Poids des différents éléments :

réservoir : $280 \mathrm{~T}$, tube : $14 \mathrm{~T}$, structure : $26 \mathrm{~T}$, gas $\mathrm{SF}_{6}: 60 \mathrm{~T}$.

L'année 1985 a été la première année de finance- ment du projet. Les travaux de bâtiment ont commencé en février 1986 et le réservoir a été installé à partir de septembre 1986 (Fig. 9). Les travaux relatifs au bâtiment et au réservoir se sont terminés durant l'été 1987 et le montage du générateur haute tension a commencé en septembre 1987. Il est prévu que le VIV délivre le premier faisceau pour les travaux de recherche au milieu de l'année 1990.

Les faisceaux du VIV seront très bien adaptés à des études «fines » liées à la structure nucléaire et focalisées sur des phénomènes de voies d'entrée (combinaisons projectile-cible) et dépendant de l'énergie de bombardement. L'énergie des faisceaux produits sera comprise entre 20 et $800 \mathrm{MeV}$, on peut citer quelques exemples de faisceaux qui seront disponibles :

\begin{tabular}{ccccccc} 
Faisceau : & ${ }^{1} \mathrm{H}$ & ${ }^{12} \mathrm{C}$ & ${ }^{32} \mathrm{~S}$ & $A=80$ & $A=130$ & $A=200$ \\
\hline Energie : & 70 & 20 & 16 & 9 & 7 & 4
\end{tabular}

(MeV/nucléon à $V=35 \mathrm{MV})$.

\section{L'avenir des machines électrostatiques.}

La réalisation du VIV avec ses nouvelles techniques de construction, constitue une étape importante 


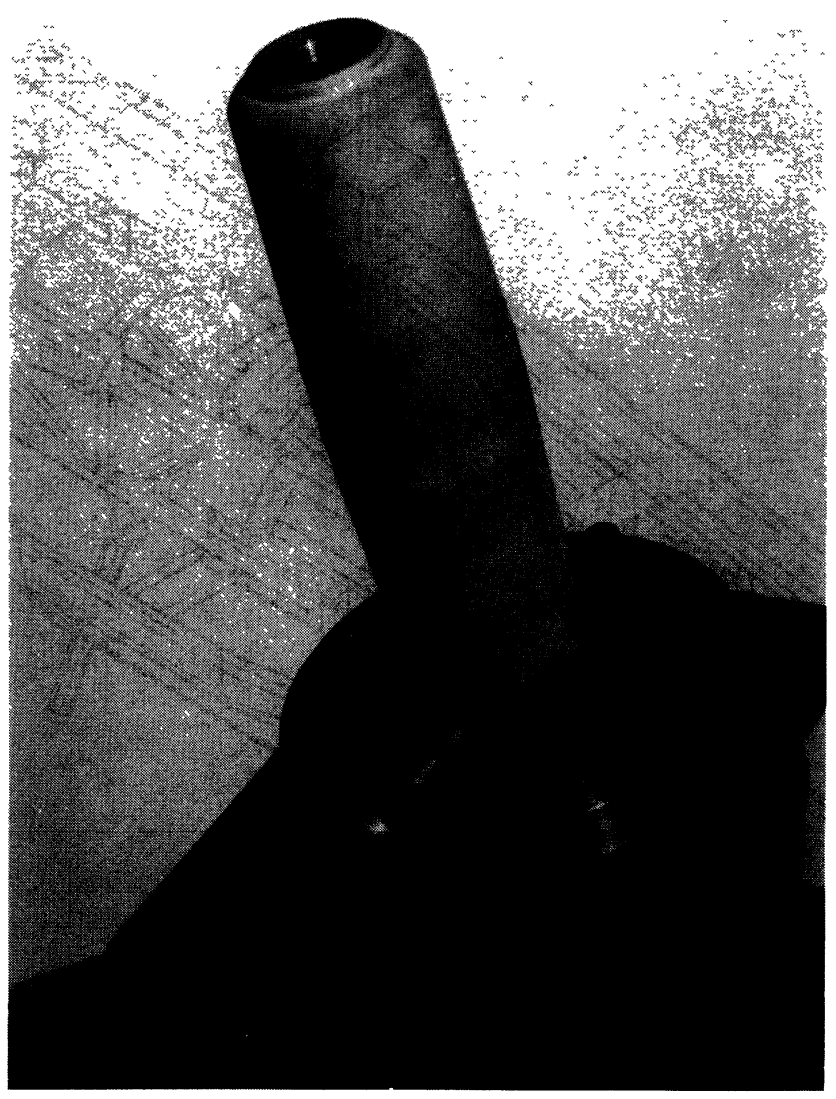

Fig. 7. - Vue d'un plot isolant utilisé dans la nouvelle machine.

[View of a post insulator used in the new machine.]
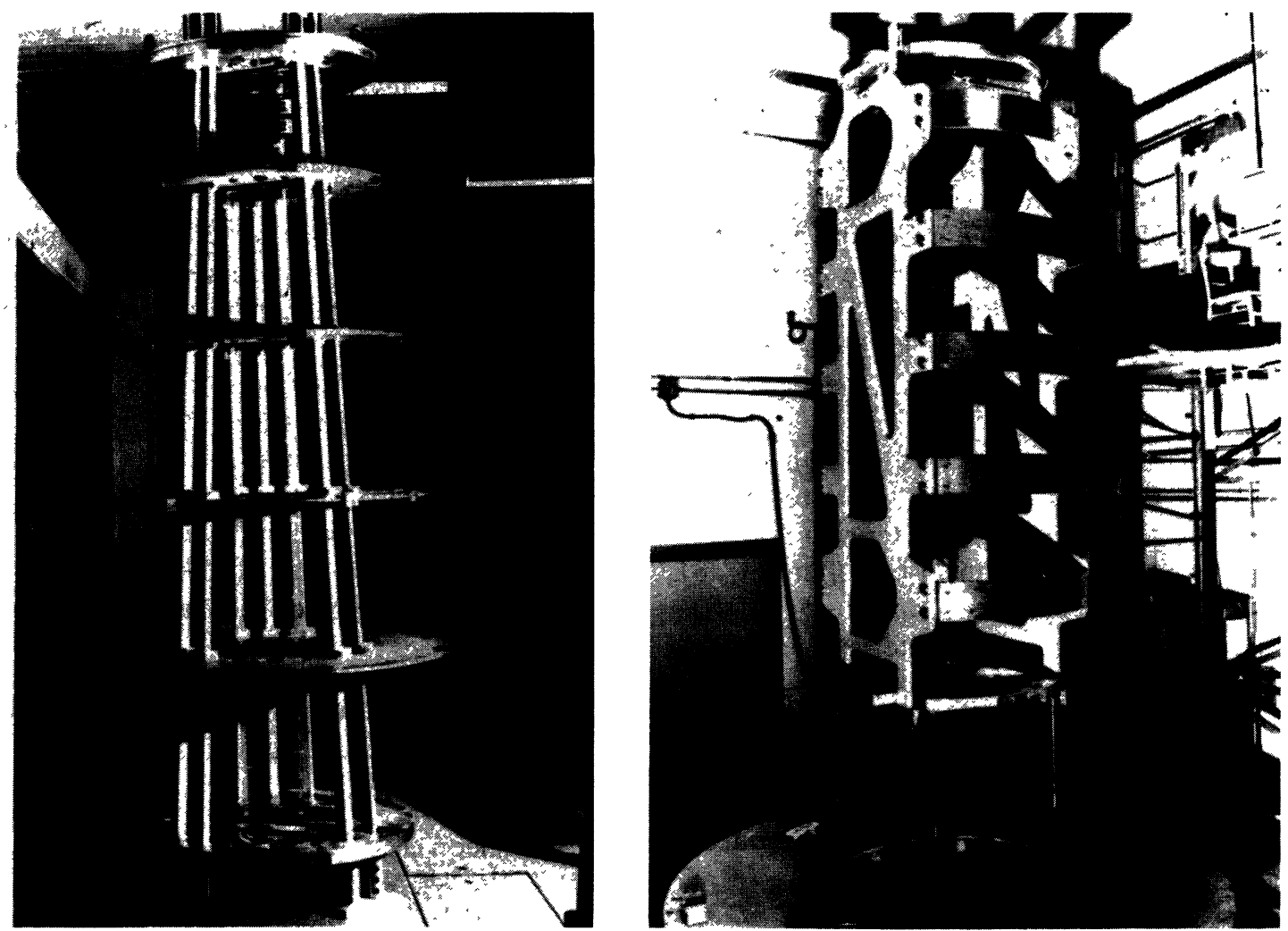

Fig. 8. - Différentes phases des essais et tests réalisés au CRN sur les isolants et sur une section du générateur haute tension.

[Different steps of the tests performed at the CRN on insulators and on one section of the high voltage generator.] 

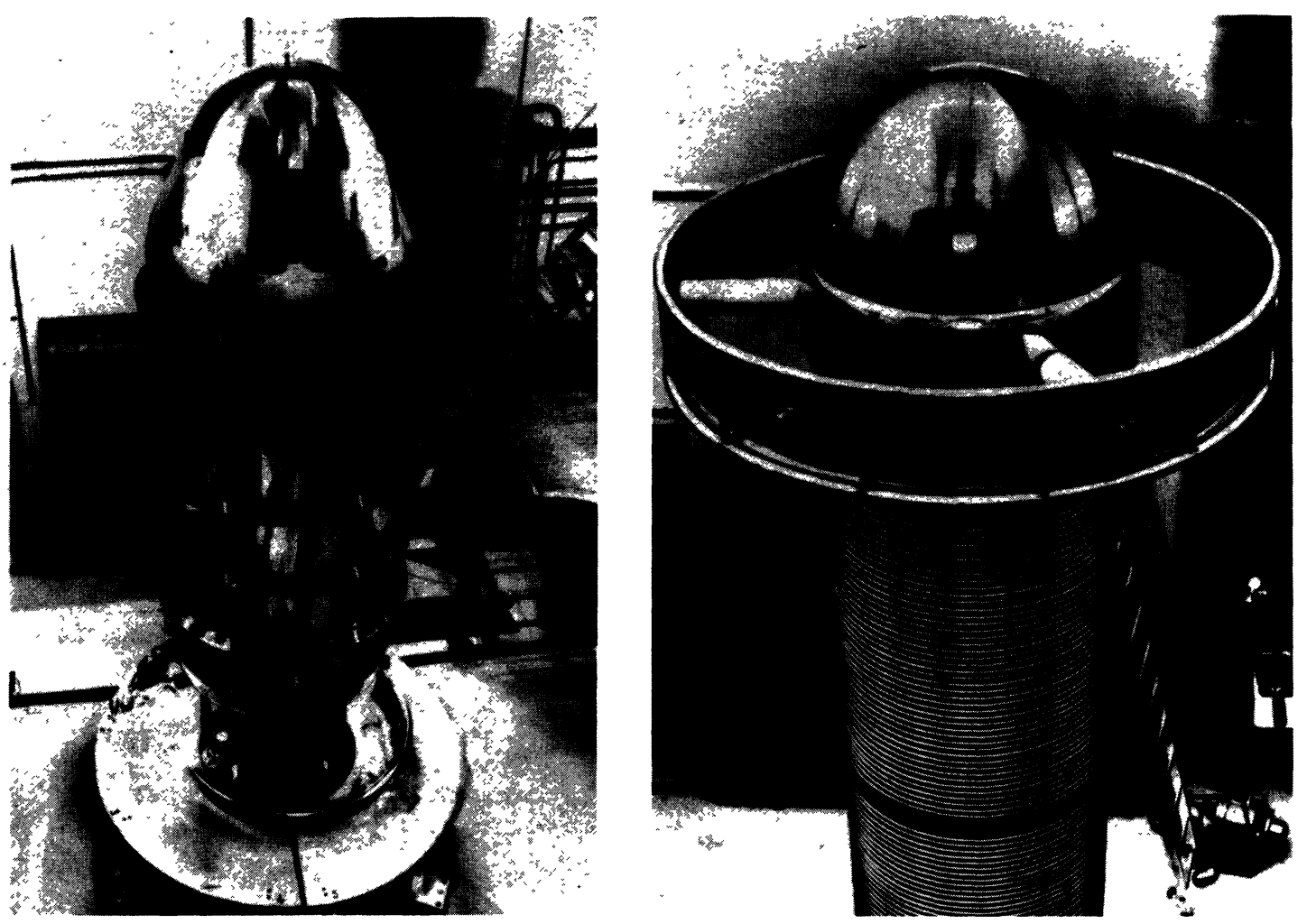

Fig. 8 (suite).

dans le développement des accélérateurs électrostatiques. Nous sommes convaincus que la réussite du projet ouvrirait la voie à une nouvelle génération de machines pour lesquelles des utilisations diverses peuvent être envisagées :

- construction de machines de quelques MV avec les techniques VIV pour de nombreuses applications dans les domaines dits «périnucléaires » où il y a actuellement une forte demande pour des faisceaux d'ions ou d'électrons ; ces machines seront plus compactes, plus fiables et aussi moins chères que les accélérateurs « classiques »;

- utilisation de l'accélérateur Tandem comme injecteur dans des projets nouveaux. Dans plusieurs laboratoires de physique nucléaire des réalisations sont en cours (Tab. I). Le Tandem peut également être utilisé comme injecteur dans des machines de faible cycle utile (synchrotron, anneau de stockage), il a été démontré (Brookhaven, Heidelberg et Oak Ridge) qu'il permet d'accélérer des faisceaux de très grande intensité $(i>100 \mu \mathrm{A})$ pendant des intervalles de temps très courts $(t<200 \mu \mathrm{s}$ et $\Delta t>20 \mathrm{~ms})$;

- utilisation de l'accélérateur Tandem et d'un faisceau d'électrons pour la réalisation d'un laser à électrons libres. Une telle installation utilisant une machine de $6 \mathrm{MV}$ est opérationnelle à l'Université de Santa Barbara (USA). Des études sont en cours à la société VIVIRAD pour un projet de plus grande envergure.

Tableau I. - Laboratoires de physique nucléaire disposant d'un système accélérateur composé de deux machines avec le Tandem comme injecteur.

[Nuclear physics laboratories having at their disposal an accelerator system composed of two machines with the Tandem used as injector.]

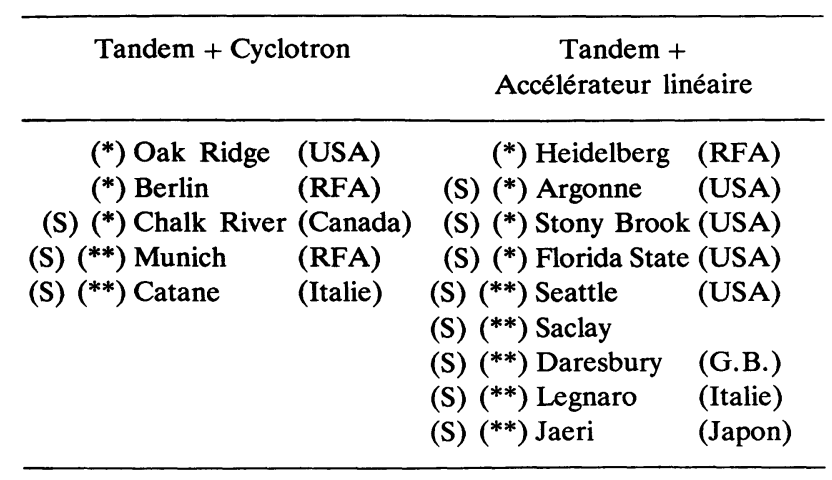

(S) : Cyclotron ou accélérateur linéaire supraconducteur.

$\left(^{*}\right)$ : en fonctionnement.

$(* *)$ : en cours de réalisation. 


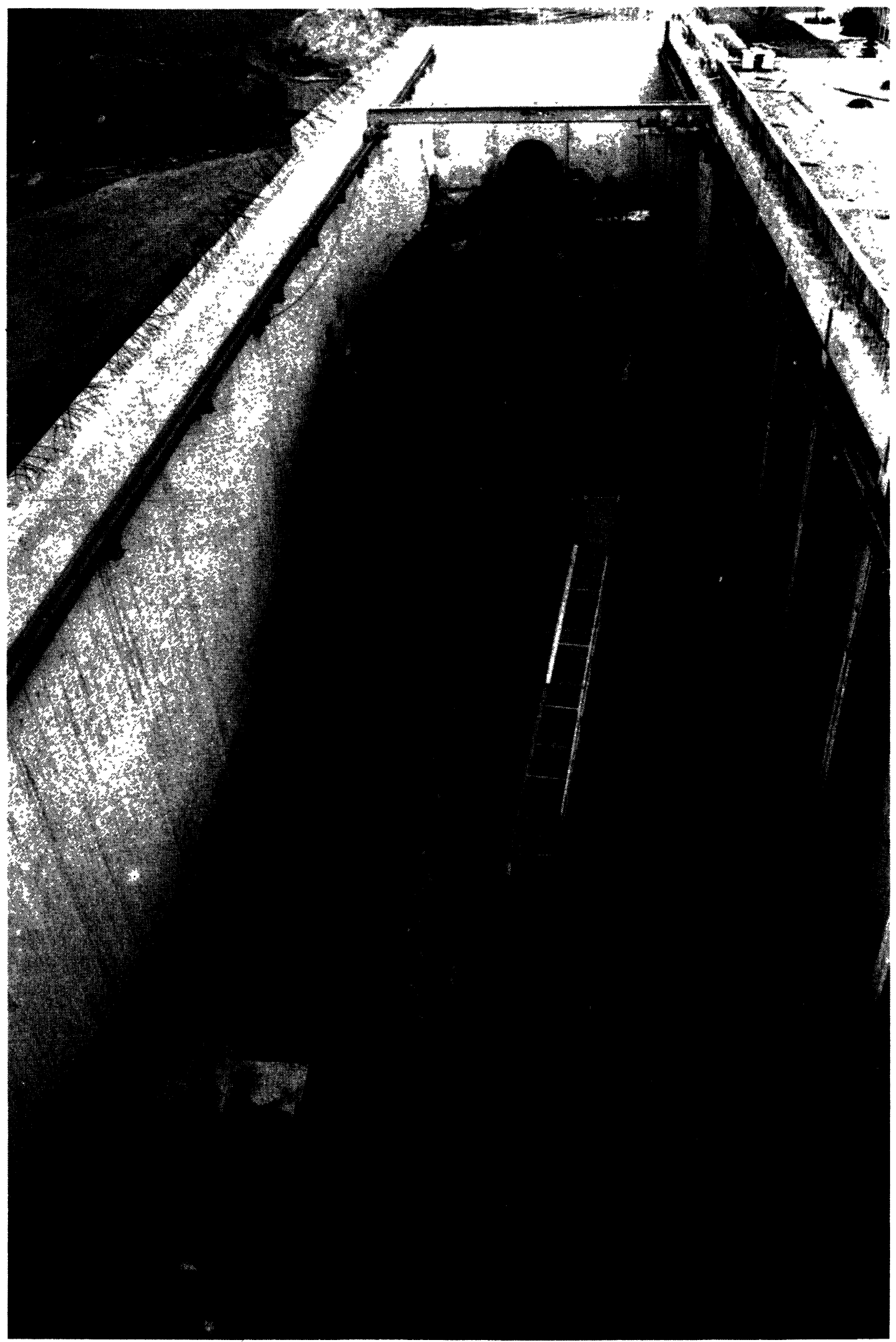

Fig. 9. - Vue du réservoir VIVITRON après assemblage in situ en mars 1987.

[View of the VIVITRON tank after the assembly was completed in March 1987.] 
Les nouvelles techniques de construction du VIV permettent de réduire sensiblement les dimensions radiales du générateur haute tension. Cependant la longueur de l'accélérateur dépend essentiellement des possibilités d'accélération du tube qui, dans le cas d'un tube de grande longueur, sont limitées actuellement à des champs de l'ordre de $20 \mathrm{kV} / \mathrm{cm}$.
Des progrès dans ce domaine constitueraient évidemment un développement majeur et nous permettraient peut-être un jour de construire la machine de rêve : un accélérateur Tandem de $50 \mathrm{MV}$ qui, conçu avec les techniques VIV et équipé d'un tube de $100 \mathrm{kV} / \mathrm{cm}$, pourrait avoir la forme d'une sphère de $10 \mathrm{~m}$ de diamètre ! 